\title{
EVALUACIÓN DE TRES MÉTODOS DE ESCARIFICACIÓN EN SEMILLAS DE ALGARROBO (Hymenaea courbaril L.)
}

\section{EVALUATION OF SCARIFICATION METHODS FOR SEEDS OF ALGARROBO (Hymenaea Courbaril L.)}

\author{
Andrés Felipe Orozco-Cardona ${ }^{1}$, Natalia Franco-Herrera², Laura Andrea Taborda-Beltrán ${ }^{2}$ \\ ${ }^{1}$ Docente Programa de Biología, Investigador Centro de Estudios e Investigaciones en Biodiversidad y Biotecnología (CIBUQ), Universidad del Quindío. \\ ${ }^{2}$ Estudiantes de IX semestre del Programa de Biología. Universidad del Quindío.
}

Fecha de recibido: Febrero 3 de 2010

Fecha de aceptado: Junio 9 de 2010

Correspondencia: Programa de Biología, Universidad del Quindío, Avenida Bolívar calle 12 norte Armenia, Quindío. Correo electrónico: andresorozco@uniquindio.edu.co

\section{RESUMEN}

Se realizó un estudio con el fin de evaluar la efectividad de tres métodos de escarificación: mecánico, físico y químico sobre la germinación de semillas de algarrobo (Hymenaea courbaril L.). Se tomaron 20 semillas por cada tratamiento, siendo 10 de ellas la réplica (200 semillas en total). Una vez aplicados los respectivos tratamientos en cada uno de los métodos evaluados, las semillas fueron puestas en recipientes plásticos con tierra y llevadas posteriormente al invernadero de la Universidad del Quindío. Un mes después se tomaron datos de porcentajes de semillas germinadas, no germinadas y muertas, encontrando que dentro de los métodos empleados, el químico mostró mejores resultados, y dentro de los tratamientos el más eficaz fue el de $\mathrm{H}_{2} \mathrm{SO}_{4}$.

Palabras claves: Escarificación, físico, químico, mecánico, semillas, germinación.

\section{ABSTRACT}

A study was carried out attempting to evaluate the effectiveness of three methods of scarification: mechanical, physical and chemical over the germination of algarrobo seeds (Hymenaea courbaril). 20 seeds were taken per treatment, being 10 of them the replay (200 seeds in total). Once applied the respective treatments in each of the evaluated methods, the seeds were put with soil into plastic containers and taken to the University of Quindio greenhouse later on. A month later, data of germinated, no germinated and dead seeds percentage were taken, finding out that among the used methods, the chemical method showed better results, and among the treatments the most effective was: $\mathrm{H} 2 \mathrm{SO} 4$.

Key words: Scarification, physical, chemical, mechanical, seed, germination.

\section{INTRODUCCIÓN}

Un gran número de semillas de especies forestales no germinan debido a que la testa dura impide la entrada de agua (latencia) y algunas semillas no germinan si no tienen un tratamiento de escarificación (1).

Varias técnicas de escarificación han demostrado su efectividad para disminuir la dureza y acelerar el proceso de germinación (2-3). Las técnicas de escarificación química, física y térmica son de especial valor para acelerar el proceso de germinación (4).

Hymenaea courbaril L., conocido comúnmente como algarrobo, presenta dificultades para su germinación, debido a una fuerte latencia, así como a la dureza de la vaina que lo recubre (5). Las semillas están envueltas en una pulpa seca de color amarillento y de olor desagradable (6). Bajo condiciones naturales, la semilla puede permanecer viable y llegar a germinar en un período de 20 días con una tasa de éxito del 40 por ciento.
Con los anteriores antecedentes y por el interés ecológico y social, se han buscado métodos para acelerar la germinación, siendo uno de éstos la escarificación, el cual ha sido probado en el mejoramiento de la germinación de especies arbóreas forestales de testa dura (7).

Uno de los principales problemas del sector forestal en Colombia es la utilización de recursos forestales sobre todo el consumo de madera en construcción, uso por compañías ferroviarias y en la industria de manufactura (8). Aunque la producción forestal no ocupa un renglón económico importante; lo cual se debe a dos razones: poca disponibilidad de materia prima y el crecimiento de la demanda interna y externa de productos finales (9).

Esta situación se ha expresado de dos formas: por un lado, la poca diversificación de los productos extraídos de los bosques, tanto a nivel de especies como de partes vegetales, y por el otro lado, el gran desperdicio generado a lo largo de todo el proceso de extracción de aprovechamiento forestal 
que se ha desarrollado en el país no permite una regeneración sostenible de los recursos sino por el contrario, propicia la deforestación masiva (9).

Según el Libro Rojo de Plantas Maderables de Colombia (8); el Algarrobo (Hymenaea courbaril L.) es una de las especies vulnerables, pero en un futuro podría estar evaluado en otras categorías. Esta es una especie de escasa reproducción natural debido a que sus semillas suelen ser fácilmente atacadas por termitas, la propagación de Hymenaea es realizada principalmente por mamíferos, monos y murciélagos que, en muchas oportunidades también son los que destruyen las plántulas cuando apenas salen a la superficie de la tierra. Su prolongado ciclo de crecimiento, así como la falta de manejo de silvicultura, el ritmo actual de la tala y la poca existencia de árboles en los bosques naturales explica su escasez y rápido deterioro (10).

La madera es de ricos colores y durable, tiene una variedad de usos, como una especie ornamental y como árbol de sombra en cafetales. Hoy en día, los árboles maderos se cosechan de rodales naturales, pero la especie es un buen prospecto para el manejo forestal (5). Esta especie tiene gran aprovechamiento forestal en diferentes ámbitos; ya que su madera es usada para la manufactura de mangos de herramienta y de cierta clase de equipos deportivos, muebles doblados al vapor y partes para botes, pisos y escalones de escaleras. Otros usos incluyen artículos torneados y artesanías, molduras interiores y vigas. La especie se recomienda para chapa decorativa, y la madera se puede usar también como una fuente de pulpa para leña y carbón (5).

A pesar de este potencial, aún no sientan un precedente legal que incentive el cultivo programado del algarrobo (Hymenaea courbaril L.), ni que impulse investigaciones para darle un mejor tratamiento comercial y productivo, pues la escasa concentración de cultivos no es suficiente para establecer mercados, por el contrario, la falta de regeneración está propagando su extinción (10).

Por lo anterior se hace necesario evaluar la efectividad de diferentes métodos de escarificación sobre la germinación de semillas de algarrobo (Hymenaea courbaril L.).

\section{MATERIALES Y MÉTODOS}

\section{Área de estudio}

El estudio se realizó en el segundo semestre del año 2009 en la ciudad de Armenia, en el invernadero de la Universidad del Quindío, el cual tiene una altitud de 1490 a 1530 m.s.n.m. con unas coordenadas geográficas: latitud norte 40 32', longitud oeste 75 $40^{\circ}$, una temperatura promedio de 19.5 oC, una precipitación $2.436 \mathrm{~mm} / \mathrm{año}$, y una humedad relativa de $65-75 \%$.

\section{Fase de Campo:}

Los frutos de algarrobo fueron colectados en el municipio de Montenegro, vereda Risaralda, finca El Amparo, con coordenadas N 4 $33^{\prime} 30.84^{\prime \prime}$ W 75 $45^{\circ} 10.92^{\prime \prime}$. La recolección de los frutos se llevó a cabo en este lugar, debido a que fue allí donde se encontraron árboles en estado de fructificación en el momento de realizar el estudio, por lo tanto garantizaban la disponibilidad del material vegetal.

Una vez identificados los individuos se procedió a bajar los frutos maduros y para esto se utilizó un bajarramas el cual permitió acceder directamente a las semillas. Posteriormente se procedió a transportarlos y para ello se emplearon bolsas de tela porosa para evitar el deterioro o posible daño en los frutos y sus respectivas semillas.

Una vez colectados, se llevaron al laboratorio de Biología de la Universidad del Quindío, donde se abrieron los frutos para extraer las semillas, para esto fue necesario el uso de un martillo, con el cual se golpearon los frutos hasta quebrarlos; para eliminar el mucílago que envuelve cada una de las semillas, fueron puestas a secar al aire libre en condiciones de sombra y posteriormente se llevó a cabo el despulpe de manera manual, con la ayuda de un cepillo.

Se tomaron 200 semillas en total; estas fueron distribuidas en grupos de 20 semillas, de tamaños uniformes entre 2,50 $\mathrm{cm}$ y 3,20 cm de largo estas medidas fueron tomadas con un calibrador digital, se tuvo en cuenta que cada semilla estuviera en buen estado (sin perforaciones), 10 de estas semillas fueron tomadas como la réplica y a cada grupo se le aplicó un tratamiento diferente (10 procedimientos en total por cada 20 semillas) (Tabla 1$)$.

Tabla 1. Tipos de tratamientos empleados para la escarificación de las semillas de $H$. courbaril.

\begin{tabular}{c}
\hline TIPO DE \\
TRATAMIENTO DESCRIPCIÓN
\end{tabular}

\begin{tabular}{|c|c|}
\hline \multirow{9}{*}{ FÍSICO* } & Las semillas fueron sumergidas en \\
\hline & $\mathrm{H}_{2} \mathrm{O}$ durante 24 horas. \\
\hline & Las semillas fueron sumergidas en \\
\hline & $\mathrm{H}_{2} \mathrm{O}$ durante 48 horas. \\
\hline & Las semillas fueron sumergidas en \\
\hline & $\mathrm{H}_{2} \mathrm{O}$ a $80^{\circ} \mathrm{C}$ durante 5 minutos. \\
\hline & Las semillas fueron sumergidas en \\
\hline & $\mathrm{H}_{2} \mathrm{O}$ a $80^{\circ} \mathrm{C}$ durante 10 minutos. \\
\hline & Las semillas fueron sumergidas en \\
\hline \multirow{6}{*}{ QUÍMICO* } & $\mathrm{H}_{2} \mathrm{SO}_{4}$ al $98 \%$ durante 5 minutos. \\
\hline & Las semillas fueron sumergidas en \\
\hline & $\mathrm{H}_{2} \mathrm{SO}_{4}$ al $98 \%$ durante 10 minutos. \\
\hline & Las semillas fueron sumergidas en \\
\hline & $\mathrm{HCl}$ al 98\% durante 5 minutos. \\
\hline & Las semillas fueron sumergidas en \\
\hline \multirow{4}{*}{ MECÁNICO } & $\mathrm{HCl}$ al $98 \%$ durante 10 minutos. \\
\hline & Las semillas fueron lijadas (lija $\mathrm{N}^{\circ}$ \\
\hline & $\begin{array}{l}\text { 100) en el lado opuesto al micrópilo, } \\
\text { hasta dejar descubierto el cotiledón. }\end{array}$ \\
\hline & $\begin{array}{l}\text { Las semillas no fueron sometidas a } \\
\text { ningún tipo de tratamiento. }\end{array}$ \\
\hline
\end{tabular}

* Luego de aplicar el respectivo tratamiento las semillas fueron

Rev. Invest. Univ. Quindío (20): 36 - 41. Armenia - Colombia 
lavadas con agua destilada.

Fase de vivero:

Después de aplicar los distintos tratamientos, cada una de las semillas fue puesta en un recipiente plástico con tierra negra no desinfectada (esto con el fin de simular condiciones naturales), debidamente rotulado con el tratamiento aplicado, estos, fueron llevados al invernadero de la Universidad del Quindío para su respectivo control y seguimiento. Las semillas se regaron con agua corriente cada tres días con ayuda de una regadera manual de jardinería.

Para el procesamiento de los datos, se tuvieron en cuenta los siguientes parámetros: porcentaje de semillas germinadas, porcentaje de semillas sin germinar y porcentaje de semillas muertas (descompuestas o atacadas por patógenos), esta información fue registrada un mes después de haber aplicado los respectivos tratamientos (4 de septiembre a 4 de octubre de 2009), sin embargo, se hicieron observaciones para determinar el tiempo (en días) y el tipo de tratamiento en donde se dio la germinación de manera más rápida.

Las semillas tomadas como no germinadas, fueron hidratadas durante 72 horas. Pasado este tiempo, fueron abiertas, dejando los cotiledones expuestos para ser sometidos a la prueba de viabilidad, la cual consiste en sumergir las semillas en una solución de 2, 3, 5 Trifenil - 2H Tetrazolium Chloride al $1 \%$, durante 24 horas, en total oscuridad a temperatura ambiente, según la intensidad de la coloración (rojo, rosado y/o incoloro) en los cotiledones, para determinar la viabilidad de cada semilla.

\section{Análisis estadístico:}

Luego de tener los datos, se realizaron ANOVAS de un factor por medio del paquete estadístico Statgraphics Centurion, para determinar si existía un método de escarificación eficaz y dentro de estos un tratamiento óptimo para la germinación de las semillas; (el porcentaje de confiabilidad empleado fue del $95 \%)$. Se realizo un diseño experimental de tipo aleatorio, es decir un diseño por cada tipo de tratamiento.

Luego de dos meses, las plántulas que presentaban una altura mayor a $15 \mathrm{~cm}$ fueron trasladadas a bolsas de polietileno, allí se llevó a cabo el proceso de lignificación y endurecimiento de las plántulas.

Después que las plántulas se consideraron como "estables" dentro del invernadero, algunas fueron llevadas a la zona donde se colectaron las semillas para ser sembradas, otras se dejaron a disposición del Centro de Estudios e Investigaciones en Biodiversidad y Biotecnología (CIBUQ) para usos de reforestación.

\section{RESULTADOS Y DISCUSIÓN}

Dentro de los tipos de escarificación empleados (físico, químico y mecánico), se encontró que el químico registra los mayores porcentajes de germinación (90\%) (figura 4) en las semillas de $H$. courbaril, mientras que el método menos eficaz fue el mecánico, donde se encontró el porcentaje de germinación más bajo (20\%) y la cantidad de semillas muertas más alto (80\%) (Figura 1).

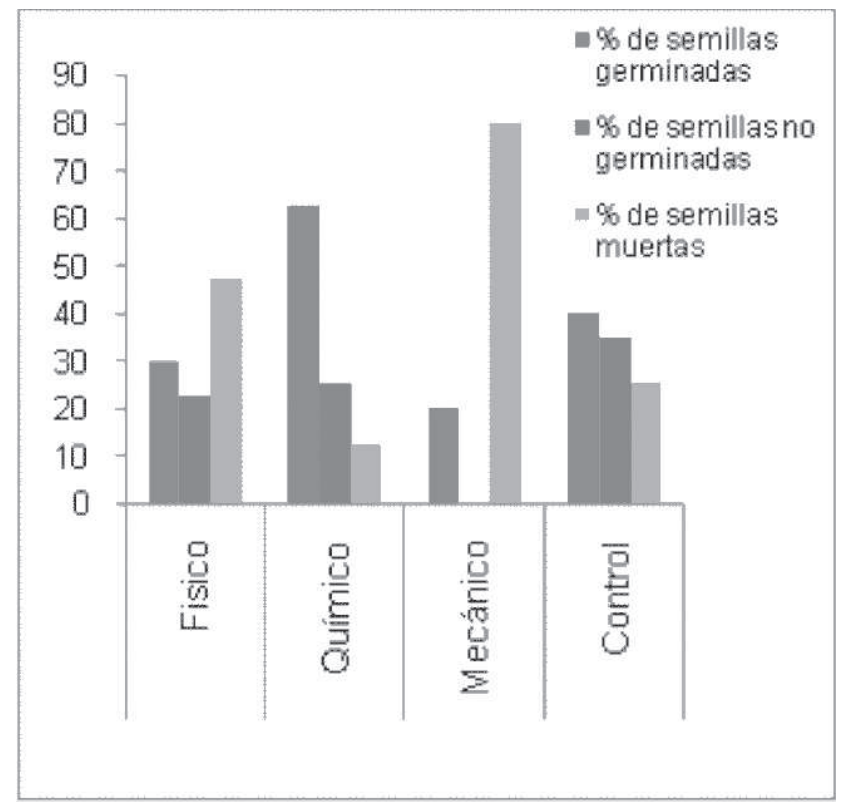

Figura 1. Porcentaje de semillas muertas, no germinadas y germinadas en los diferentes tipos de escarificación.

Las primeras semillas germinadas se registraron a los 15 días; esto ocurrió para las tratadas con $\mathrm{H}_{2} \mathrm{SO}_{4}$ durante 5 minutos.

Para el tratamiento control, el $40 \%$ de las semillas germinaron en un periodo de 30 días; el $35 \%$ de las semillas no germinaron y el $25 \%$ restante fueron semillas muertas (Figura 2). Según Francis (1990) (5), las semillas germinan en un período de 20 a 30 días bajo condiciones naturales con una tasa de éxito del 40 al $90 \%$, lo cual ratifica los resultado encontrados.

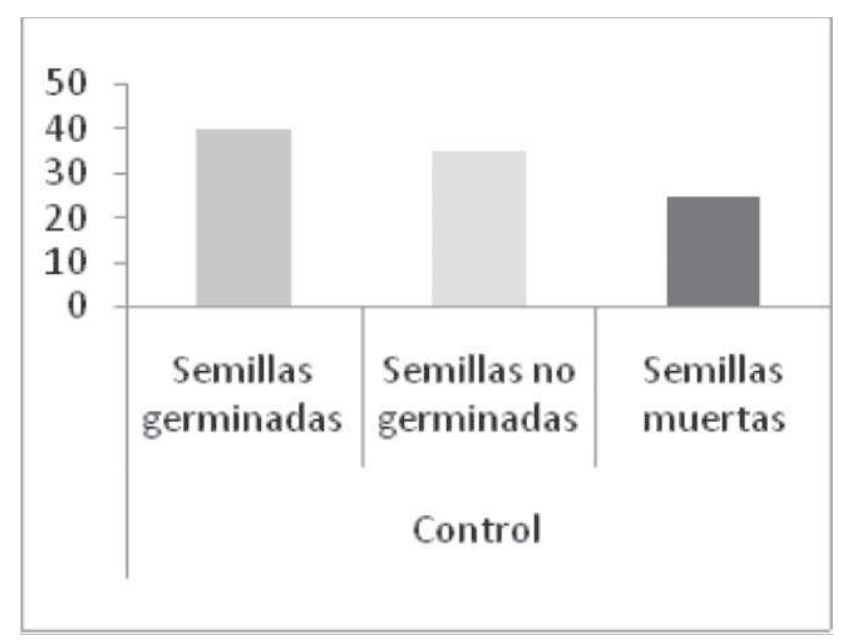

Figura 2. Porcentaje de semillas germinadas, no germinadas y muertas en el tratamiento de control.

Dentro de los tratamientos físicos, las semillas que fueron puestas a imbibir durante 24 horas en agua a temperatura ambiente presentaron un mayor porcentaje de germinación 
del $80 \%$ (Figura 3), Torres et al. (1996) (11), obtuvieron porcentajes de germinación en P. laevigata de alrededor del $64 \%$ sometiendo las semillas a tratamientos pregerminativos de remojo en agua a temperatura ambiente por 24 y 36 horas. Según Welbaum et al. (1998) (12), plantean que los tratamientos de hidratación parcial incrementan el establecimiento, debido a que aceleran la emergencia de las plántulas y disminuye la perdida de electrolitos por las semillas (aminoácidos y azucares), esto último contribuye a reducir los ataques fúngicos considerablemente.

Niembro (1979) (13), menciona al respecto, que los remojos permiten acelerar los procesos de hidrólisis (descomposición de los procesos químicos por acción del agua) de las reservas del embrión y por lo tanto se acelera la germinación de la semilla, lo cual se confirma en este trabajo bajo el tratamiento de imbibición por 24 horas(Figura 3).

En el estudio de Prosopis tamarugo el mejor tratamiento de escarificación de semillas fue el de agua a temperatura ambiente por 48 horas (14). Aunque para nuestros resultados el porcentaje de semillas no germinadas fue el más alto para aquellas sumergidas durante este tiempo (48 horas) (Figura 3).

La disminución en la germinación de semillas cuando se someten a hidratación podría deberse a la falta de estandarización de los tratamientos pre-germinativos para el lote de semillas empleado, lo que debe cumplirse para obtener los mejores resultados con la aplicación de dichos tratamientos (15). Otra causa de la muerte de las semillas puede ser el remojo prolongado de estas, dado que la imbibición impide la aireación y la semilla muere por asfixia o bien por la exosmosis (difusión u osmosis del interior hacia afuera a través de las paredes) de enzimas y nutrimentos (16).

Sin embargo, los tratamientos antes mencionados mostraron mejores resultados que los obtenidos cuando las semillas fueron sumergidas en agua a una temperatura de $80^{\circ} \mathrm{C}$, ya que para los 2 tiempos ( 5 y 10 minutos), se encontraron tasas de germinación demasiado bajas, incluso llegando a 0 (cero) (Figura 3).

Según Francis, (1990) (5) sumergir las semilla en agua caliente durante 25 y 30 segundos se puede obtener un porcentaje de germinación entre 90 y $93 \%$, respectivamente, al igual que el estudio de Sánchez, (2002) (15) donde encontró que las semillas de L. leucocephala sometidas a una escarificación en agua a 100 y 80 oc de temperatura reportan los mayores valores de germinación. Para $H$. courbaril los porcentajes de germinación para el tratamiento con agua a $80^{\circ} \mathrm{C}$ en ambos tiempos (5 y 10 minutos), se encontraron tasas de germinación muy bajas, llegando a cero . De manera general se puede señalar que la inmersion de las semillas en agua caliente no ofrece ventajas en relación al control igual a lo encontrado por Sanabria et al. (2001) (4) en semillas de C. rotundifolium. Niembro (1979) (13), señala que con temperaturas elevadas se incrementa la tasa metabolica y se destruyen algunas enzimas, lo cual causa el deterioro de la semilla. Ademas se deben determinar correctamente la temperatura del agua y el tiempo de inmersión si se quieren obtener buenos resultados, el mismo autor señala que tiempos de inmersión prolongados en agua caliente ocasionan, por lo general, daños irreversibles en las semillas, con efectos drásticos en la germinación (16).

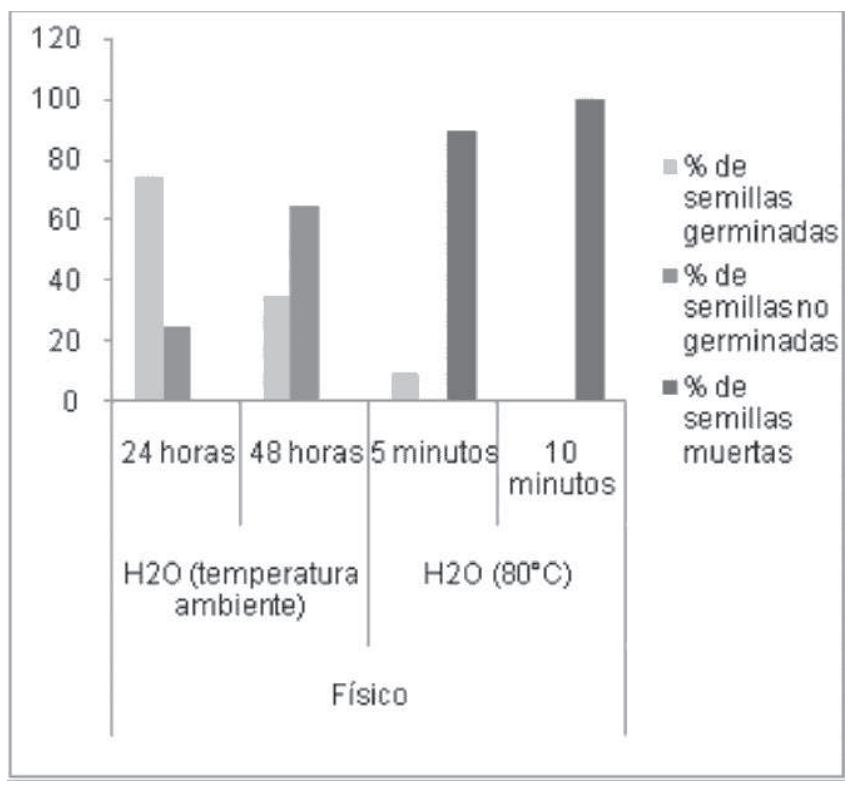

Figura 3. Porcentaje de semillas muertas, no germinadas y germinadas en los diferentes tratamientos dentro del tipo de escarificación física.

Uno de los tratamientos que a criterio de Loaiza (1979) (17), resulta muy significativo para la escarificación de semillas de leguminosas y otras especies de testa dura son los tratamientos químicos, los cuales incluye pruebas con ácidos (sulfúrico, nítrico y fosfórico). Pruebas de germinación en Puerto Rico, sugieren que la escarificación de las semillas mediante muescas o el baño en ácido sulfúrico concentrado ha aumentado el porcentaje y reducido el tiempo de germinación (5). En esta investigación se obtuvo que las semillas tratadas con ácido sulfúrico durante 5 minutos fueron las que presentaron el porcentaje de germinación más alto $(90 \%)$, la tasa de semillas no germinadas más baja (0\%) y el tiempo de germinación más corto (15 días) (Figura 4). Estos resultados concuerdan con los publicados por Pietrosemoli \& Mendiri (1997) (18) quienes reportan como mejor método de escarificación el $\mathrm{H}_{2} \mathrm{SO}_{4}$ por 5 y 8 minutos en Clitorea ternatea obteniendo valores de germinación de 59 y $53 \%$ respectivamente.

Los resultados obtenidos en las semillas de algarrobo sumergidas en ácido clorhídrico no fue eficiente en ninguno de los tiempos, ya que la tasa de germinación y de semillas no germinadas fueron muy similares (Figura 4). Caso contrario ocurrió en el trabajo realizado por Godínez (1991) (19) quien estudió en ocho especies de cactáceas el efecto del ácido clorhídrico a diferentes concentraciones y observó que la inmersión en el ácido le permitió obtener porcentajes de germinación altos. En contraste Álvarez \& Montaña (1997) (20) observaron que la inmersión de semillas en ácido

Rev. Invest. Univ. Quindío (20): 36 - 41. Armenia - Colombia 
clorhídrico de Cephalocereus chrysacanthus, Cephalocereus hoppenstedtii, Ferocactus latispinus, Stenocereus stellatus y Wilcoxia viperina, no influyó de manera significativa en los porcentajes de germinación.

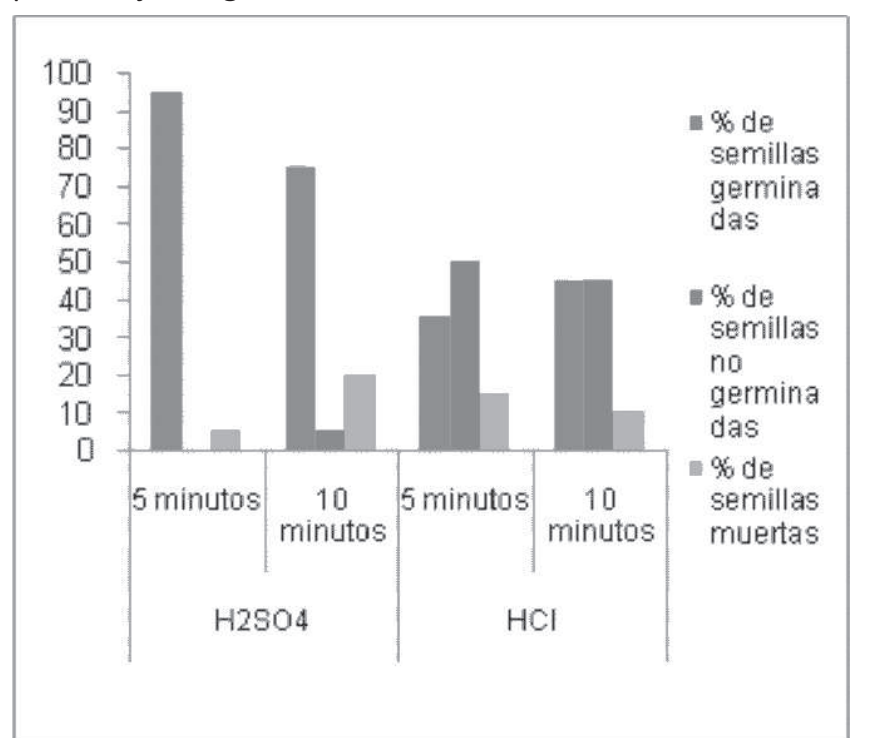

Figura 4. Porcentaje de semillas muertas, no germinadas y germinadas en los diferentes tratamientos dentro del tipo de escarificación química.

La escarificación mecánica con lija se ha empleado con éxito en semillas de Prosopis cineraria, Leucaena leucocephala y Acacia nilotica (21); igual a lo encontrado por Aubeterre et al. (2006) (22) para semillas de cinco especies de cactáceas, donde los tratamientos más efectivos fueron los mecánicos (lijados a 5 y 10 min.) con una germinación de 83,2 y 75,2\% respectivamente. En nuestra investigacion el tratamiento mecánico fue el menos eficiente; ya que las semillas muertas eran 4 veces mayor que las germinadas (80 y $20 \%$ respectivamente), confirmandose así como el método menos práctico para realizar en este tipo de semillas (Figura 5). Las semillas con testa dañada (para este caso deterioradas por lijado) son mas suceptibles a la fuga de cantidades sutanciales de solutos (azúcares y ácidos orgánicos). La fuga se suspende después de un corto periodo, en pocos minutos o una cuantas horas como máximo en semillas vivas y saludables, probablemente debido a la reparación de membranas dañadas. Las sustancias perdidas forman la base para el crecimiento activo de hongos (23), lo cual se vio reflejado en esta investigación, pues se encontraron semillas completamente descompuestas.

La prueba de viabilidad o prueba de tetrazolio (TZ), se realizó en las semillas no germinadas con el fin de saber la viabilidad de germinación, esta se determina en función del patrón de tinción del embrión y la intensidad de la coloración, para lo cual se encontró que la coloración en la mayoría de las semillas era rosa, mostrando así que estas semillas eran parcialmente viables (24), esto puede indicar la presencia de un tegumento muy impermeable, lo cual retrasa la imbibición y por consiguiente la germinación de las semillas (4).

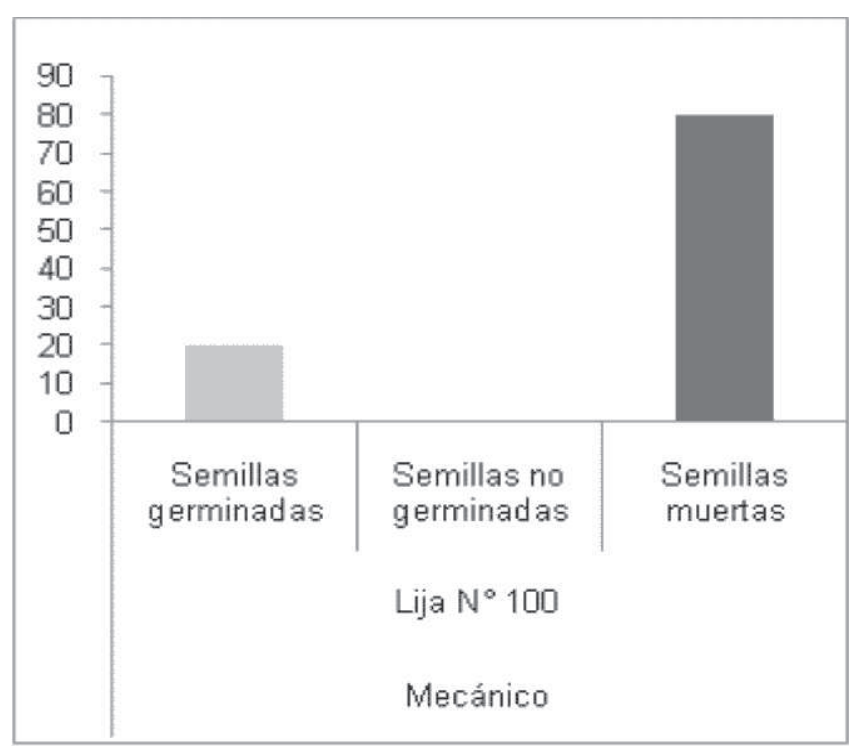

Figura 5. Porcentaje de semillas germinadas, no germinadas y muertas en el tipo de escarificación mecánica.

Tabla 2. Resultados obtenidos a partir del ANOVA de un factor.

\begin{tabular}{l|c|c}
\hline \multicolumn{1}{c|}{ VARIABLE } & P-valor & F-ratio \\
\hline Tipo de & 0,1417 & 2,09 \\
escarificación & $0,0008^{*}$ & 8,30 \\
\hline
\end{tabular}

*existen diferencias significativas

Los datos obtenidos para ANOVA muestran que no hay diferencias significativas entre los tipo de escarificación (Tabla 2), aunque en contraste con las gráficas se obseva lo contrario, siendo los valores de las semillas germinadas diferentes para cada tipo de escarificación aplicado, mientras que si existen diferencias significativas entre los tratamientos aplicados.

\section{CONCLUSIONES}

$\checkmark$ El método de escarificación químico con ácido sulfúrico es el más eficaz para aumentar el porcentaje de germinación de semillas de Hymenaea courbaril.

$\checkmark$ La dormancia física impuesta por la cubierta de la semilla puede ser superada por este tipo de tratamientos pregerminativos (escarificación), los cuales garantizan una óptima forma para reducir el tiempo y aumentar el porcentaje de germinación.

$\checkmark$ A pesar del gran potencial del algarrobo, las autoridades del mundo aún no sientan un precedente legal que incentive el cultivo programado de Hymenaea courbaril, ni tampoco que impulse investigaciones para darle un mejor tratamiento comercial y productivo. Por ello se requiere 
que las autoridades ambientales, las universidades y las empresas madereras propicien investigaciones como estas para proteger la especie y poder así conformar un paquete tecnológico forestal a fin de promover su conocimiento y regular su manejo, aprovechamiento y comercio.

\section{AGRADECIMIENTOS}

Los autores del proyecto agradecen al Programa de Biología y al Centro de Estudios e Investigaciones en Biodiversidad y Biotecnología de la Universidad del Quindío (CIBUQ) por su apoyo logístico para realizar esta investigación.

\section{BIBLIOGRAFÍA}

1. Poulsen, K; \& Stubaard, F; 2000. Técnicas para la escarificación de semillas forestales. Centro agronómico tropical de investigación y enseñanza (CATIE) 36:60.

2. Burbano, E; 1990. Efecto de la escarificación química y el almacenamiento en la calidad de semilla de Centrosema. Revista Pasturas Tropicales. 12(13): 11-55.

3. Fariñas, D; Sanabria, D; \& Silva, R. 1997. Escarificación química de tres especies de Centrosema para sabanas bien drenada. Revista Zootecnia Tropical. 15(2): 221-237.

4. Sanabria, D; Silva, R; Oliveros, M; \& Barrios, R; 2001. Escarificación química y térmica de semillas subterráneas de Centrosema rotundifolium. Revista Bioagro 13(3):117-124.

5. Francis, J; 1990. Hymenaea courbaril L. Algarrobo. Department of Agriculture, Forest Service, Southern Forest Experiment Station. 279-283.

6. Herrera, S. 2009. Árboles de la Universidad del Valle. Programa editorial Universidad del Valle. 169-170.

7. Jiménez, E; 2004. Sistema de escarificación de semillas de tagua (Phytelephas aecuatorialis) para mejorar la germinación. Revista Tecnológica. 17(1): 46-54.

8. Libro Rojo de Plantas de Colombia. 2006. Especies maderables amenazadas I parte. Editado por: Cárdenas, D. y Salinas, N.

9. Informe Nacional sobre el Estado de la Biodiversidad. 1997. Tomo II: Causas de pérdida de Biodiversidad. Editado por: Chávez, M. y Arango, N. Bogotá: 3:223

10. Rojas, A; 2002., Algarrobo: una especie doble propósito. Revista del Mueble \& la Madera 55: 10-15

11. Torres, S.; Martínez, O.; García, A. \& Frias, J. 1996. Escarificación hídrica de semilla de Mezquite (Prosopis laevigata HUMB. \& BONPL. Ex. WILD M. C. JOHNST). En: Aubeterre R., Principal J. y García J. 2002. Efecto de diferentes métodos de escarificación sobre la germinación de tres especies del género Prosopis. Revista Científica 12 (2): 575-577.

12. Welbaum, E., Shen, Z., Oluoch, O. \& Jett, W. 1998. The evolution and effects of priming vegetable seed. Seed Technology 20:209-235. En: Sánchez, A. Muñoz, C., Hernández, L., Montejo, L., Suarez, G. \& Torre-Arias, Y. 2006. Tratamientos robustecedores de semillas para mejorara la emergencia y el crecimiento de Tricospernum mexicanum, árbol tropical pionero. Agronomía costarricense 30(1): 7-26

13. Niembro, A. 1979. Semillas forestales. En: convención centroamericana de semillas forestales. Ciguatepeque. Memorias, departamento de bosquesUACH: $86 p$

14. Aubeterre R., Principal J. y García J. 2002. Efecto de diferentes métodos de escarificación sobre la germinación de tres especies del género Prosopis. Revista Científica 12 (2): 575-577.

15. Sánchez A. 2002. Efecto del tratamiento con agua caliente e imbibición sobre la germinación de semillas de L. leucocephala. Revista Científica 12 (2): 581-583.

16. Niembro, A. 1989. Semillas de plantas leñosas. Ed.: Limusa, México D.F. 93 p.

17. Loaiza H. 1979, Silvicultura I, Loja Ecuador, Departamento de Publicaciones Universidad Nacional de Loja. En: Jiménez, E; 2004. Sistema de escarificación de semillas de tagua (Phytelephas aecuatorialis) para mejorar la germinación. Revista Tecnológica. 17(1): 46-54.

18. Pietrosemoli, S. \& Mendiri, J. 1997. Respuesta a la escarificación de semillas de Clitorea ternatea L. Archivos Latinoamericanos de Producción Animal. 5 (1): 28-29. En: Aubeterre R., Principal J. y García J. 2002. Efecto de diferentes métodos de escarificación sobre la germinación de tres especies del género Prosopis. Revista Científica 12 (2): 575-577.

19. Godínez, H.1991. Propagación de Cactáceas por semilla: una experiencia para su cultivo y conservación. Tesis de Licenciatura. Facultad de Ciencias. Universidad Autónoma de México. En: Navarro C. \& González M. 2007. Efecto de la escarificación de semillas en la germinación y crecimiento de Ferocactus robustus (Pfeiff.) Britton \& Rose (Cactaceae). Zona s Árida s 11(1): 195-205.

20. Álvarez, G. \& Montaña, C. 1997. Germinación y supervivencia de cinco especies de Cactáceas del Valle de Tehuacán: implicaciones para su conservación. Act. Bot. Mex. 40:43-58. En: Navarro C. \& González M. 2007. Efecto de la escarificación de semillas en la germinación y crecimiento de Ferocactus robustus (Pfeiff.) Britton \& Rose (Cactaceae). Zonas Áridas 11(1): 195-205.

21. Yepes, F. \& Arboleda, M. 2009. Promoción de la emergencia de urape (Bauhinia monandra Kurs) y retama (Thevetia peruviana (Pers) Schum), especies. Bioagro 21(1): 15-22

22. Aubeterre, R., Piñero, Z., García, E. y Figarella, M. 2006. Efecto de diferentes métodos de escarificación sobre la germinación de cinco especies de cactáceas (Opuntia picus indica, Pilosocereus moritzianus, Stenocereus griseus, Cereus deficiens y Cereus hexagonus) del Estado Lara. Simposio Taller: Experiencias en Agroforestería ejecutadas o en proceso por el Instituto Nacional de Investigaciones Agrícolas (INIA). Maracay. 13-17

23. Jara, L. 1996. Biología de semillas forestales. Centro agronómico tropical de investigación y enseñanza (CATIE) 36:32.

24. Ruiz, M. 2009. El análisis de tetrazolio en el control de calidad de semillas: Caso de estudio: cebadilla chaqueña. Instituto Nacional de Tecnología Agropecuaria (INTA).Publicación Técnica 77:2-19.

Rev. Invest. Univ. Quindío (20): 36 - 41. Armenia - Colombia 